\title{
Discours, style, format : contraintes et niveaux de structuration de la textualité des Tweets de Mouloud
}

\author{
Longhi, Julien \\ Université de Cergy-Pontoise \\ Centre de Recherche Textes et Francophonies (CRTF-EA 1392) \\ Pôle Langage Société Communication Didactique \\ julien.longhi@u-cergy.fr
}

\section{Introduction}

Dans le but de faire émerger certains aspects constitutifs des productions textuelles, nous nous attachons à décrire un corpus qui manifeste intrinsèquement une forme d'hétérogénéité. Les Tweets de Mouloud constituent une rubrique du magazine féminin $B e$, écrite chaque semaine par le chroniqueur Mouloud Achour. Dans cette rubrique, il rend compte de l'actualité sous forme de dix Tweets. Il doit ainsi se conformer à un cadre très strict (140 caractères en théorie, même s'il dépasse parfois légèrement), dont le résultat deviendra une rubrique dans un magazine hebdomadaire, tout en manifestant un style qui lui est propre. Nous avons constitué un corpus de 50 tweets (parus donc dans cinq éditions distinctes), et en proposons ici une analyse dont l'ambition est de mesurer la portée des facteurs constitutifs de ces écrits sur leur contenu, leur forme et leur organisation. Ce faisant, nous pourrons saisir avec un même objet d'étude le fonctionnement d'un genre de discours, d'un style, et d'un format d'écriture, et la manière dont ces concepts s'organisent dans la manifestation de la textualité. Des hypothèses sur la textualité et sa matérialité pourront donc être fournies par la confrontation de ces niveaux d'analyse, et offrir des interactions avec la linguistique du discours (à partir des relations notamment entre formation discursive et style ou format).

\section{Les paramètres de l'analyse}

Dans cette première section, nous décrivons les niveaux qui vont déterminer la textualité. Dans ce cas des Tweets de Mouloud, nous en voyons trois distincts : le discours, le style, et le format d'expression.

\subsection{Le discours}

Selon Sarfati (1997), un discours se caractérise par son positionnement (défini comme sa situation sociologique relativement à un groupe social donné), la qualité de son support médiatique (inscription), et l'intertextualité (le régime de relations qui règlent les rapports que les textes entretiennent entre eux ou avec d'autres textes d'un autre type de discours). La rubrique à laquelle nous nous intéressons appartient à un magazine hebdomadaire féminin. Sur son site internet, dans la page « abonnement », le magazine est décrit ainsi

\begin{abstract}
Chic, pop et sexy... Chaque vendredi, Be, c'est un vrai point de vue actu, mode et beauté ! Retrouvez chaque semaine l'actu décryptée par les Bees, toutes les tendances mode, les astuces et conseils beauté, les news de vos stars préférées et les dernières sorties culture (ciné, musique, livres...). Découvrez les meilleurs looks, les bons plans shopping en ligne et ne manquez pas les témoignages de Be for Me !
\end{abstract}

Le discours se veut moderne et dynamique, et s'adresse à la «Now generation ». Il se distingue ainsi d'autres titres tels que Elle ou Marie-Claire en ce qu'il vise un public légèrement plus jeune (les trentenaires $^{1}$ ). Il présente également des rubriques dites «people» sans être malgré tout proche des magazines de ce type. L'actualité est très présente, et c'est une autre spécificité de magazine, qui consacre 
une partie de chaque édition à des analyses de l'information, comme on peut en trouver dans Paris Match par exemple, mais avec un ton que l'on pourrait qualifier de plus léger. C'est dans cette direction que la rubrique Les Tweets de Mouloud s'inscrit: parler de l'actualité de manière humoristique, légère, ou décalée.

Pour revenir à la définition du discours par Sarfati, nous pourrions donc dire, en ce qui concerne la rubrique Les Tweets de Mouloud, que le positionnement est relatif à un certain public féminin (30-40 ans), son support médiatique est le magazine féminin hebdomadaire, et son intertextualité concerne essentiellement le discours politique. À ces éléments macro-structurants, qui conduiront à un certain nombre de normes sémantiques (Rastier, 2000) doivent s'ajouter d'autres paramètres.

En outre, une prise en compte de la notion de formation discursive peut se révéler éclairante, puisqu'elle prolonge ce qui vient d'être dit. En effet, pour Pêcheux, une formation discursive est « ce qui, dans une formation idéologique donnée, c'est-à-dire à partir d'une position donnée dans une conjoncture donnée déterminée par l'état de la lutte des classes, détermine « ce qui peut et doit être dit (articulé sous la forme d'une harangue, d'un sermon, d'un pamphlet, d'un exposé, d'un programme, etc.) » (Pêcheux, 1975 : 144). Même si Pêcheux ne parle pas de genre ou de type de texte, nous pouvons en voir une certaine trace dans cette mention. En outre, Pêcheux donne une place centrale à l'idéologie, mais on peut néanmoins retranscrire cette formulation à propos de notre corpus : ce qui est écrit par Mouloud Achour peut être mis en rapport avec une certaine position (liée au positionnement socio-historique de l'auteur, nous le verrons dans la suite), et se transpose dans une forme particulière adaptée à ce qui peut et doit être dit, dans notre cas la forme de tweet. La notion d'interdiscours est introduite par Pêcheux pour indiquer les rapports de force entre formations discursives, et également pour penser leur existence de manière dialectique. Là encore l'interdiscours prolonge le repérage du régime d'intertextualité mentionné par Sarfati, en pointant davantage encore le fait que le rapport entre les discours entraîne également une mise en rapport de positionnements, entre des types de discours spécifiques pour les tweets et le discours politique, et des normes sémantiques propres à chaque forme. Pour notre corpus, il sera intéressant de voir dans quelle mesure ce positionnement, et la ou les forme(s) qui s'y articule(nt), s'atteste dans un ou des style(s) particulier(s). Ceci posera concrètement la question de l'individualisation d'une formation discursive, à la suite de Foucault, qui précise qu'une formation discursive sera individualisée si on peut définir le système de formation des différentes stratégies qui s'y déploient : «Une formation discursive [...] détermine une régularité propre à des processus temporels; elle pose le principe d'articulation entre une série d'événements discursifs et d'autres séries d'événements, de transformations, de mutations et de processus » (Foucault, 1969 : 98-99).

\title{
2.2 Style et format : Mouloud Achour et Twitter
}

La complexité du corpus tient au fait que l'auteur de la chronique, Mouloud Achour, a une forte personnalité et un style particulier. Voici quelques informations le concernant, issues de la page Wikipedia qui lui est consacrée :

\begin{abstract}
Mouloud Achour, né 1er août 1980 à Noisy-le-Sec, est un animateur de télévision, journaliste, musicien et scénariste français. Il est chroniqueur dans l'émission Le Grand Journal de Canal+ depuis la rentrée 2008, tenant une chronique sur les nouveaux mouvements. Il participe également à la Bataille Musicale du vendredi, face à Tania Bruna-Rosso et depuis septembre 2010 le Daily Mouloud dans Le Grand Journal. Il intervient dans l'émission d'Arthur, Les Enfants de la télé, en tant que DJ Mouloud. Il a aussi été animateur sur la radio parisienne Fréquence Paris Plurielle et sur MTV. Avant le Le Grand Journal, il tenait une chronique dans La Matinale, appelée L'Avis de Mouloud. Il est aussi journaliste dans la presse écrite : il a ainsi participé à The Source, magazine spécialisé dans le hip-hop, et collabore actuellement à Technikart. Il a fondé le label Kerozen avec le groupe de rap La Caution.
\end{abstract}

Il s'agit donc d'un auteur possédant à la fois une liberté de ton, une expression peu contrainte, et une culture urbaine et contemporaine forte. Il s'engage en outre sur des sujets tels que le racisme ou les dérives de la politique, ce qui peut s'expliquer pour partie par ses origines (qu'il mentionne parfois, 
notamment avec une certaine auto-dérision). L'éthos qu'il se constitue dans le discours pourra être polémique, comme nous le verrons. En cohérence avec cela, le mode d'expression utilisé dans ce magazine est le tweet ${ }^{2}$ :

\begin{abstract}
Twitter est un service de microblogage, permettant aux utilisateurs de bloguer grâce à des messages courts (140 caractères maximum, soit une ou deux phrases). Outre cette concision imposée, la principale différence entre Twitter et un blog traditionnel réside dans le fait que Twitter n'invite pas les lecteurs à commenter les messages postés. Le slogan d'origine de Twitter, What are you doing? le définissait comme un service permettant de raconter ce qu'on fait au moment où on le fait. Prenant acte de l'utilisation du service pour s'échanger des informations et des liens, Twitter le remplace par What's happening? («Quoi de neuf? » ou encore « Que se passe-t-il ?» dans la version française).
\end{abstract}

Ce format permet d'exprimer habituellement des éléments d'actualité de manière brève et concise. Il permet également de «tweeter» des liens vers des sites que l'auteur du tweet recommande (souvent un billet sur un blog, un article de presse). Mouloud Achour est un utilisateur intensif des réseaux sociaux et des nouveaux moyens de communication, comme Twitter ou Facebook. Sur ces réseaux, il est suivi par de nombreux «followers » (111583 abonnés à son compte sur Twitter) ou ou personnes qui « aiment» (44 435 sur Facebook).

Détourné de sa fonction, le tweet utilisé dans le cadre d'une chronique pourra parfois s'apparenter à d'autres formes brèves, comme la maxime ou la sentence. Certains autres tweets, également caractérisables par leur brièveté, se présentent sous une forme binaire, avec un fait dont découle une conclusion, s'approchant ainsi du proverbe. Il est à noter que l'auteur dépasse parfois les 140 caractères, tout en restant dans des formes d'expression très brèves.

\title{
3 Présentation du corpus et thématiques : prégnance du discours et empreinte du style
}

Le corpus que nous avons constitué comprend cinq rubriques des Tweets de Mouloud, soit 50 tweets qui correspondent à des fragments ne devant pas dépasser 140 caractères, comme :

11 Selon le calendrier musulman, nous sommes en 1432. Qui a dit que les Arabes ont fait la révolution sur le tard?

Le premier pas pour aborder ces tweets nous semble être leurs thématiques. Nous avons numéroté chacun de ces tweets et les avons classés par thème (certains pouvant appartenir à deux thèmes), et avons obtenu la répartition suivante ${ }^{3}$ : 


\begin{tabular}{|l|l|l|}
\hline Catégorie & Sous-catégorie éventuelle & Twitts \\
\hline & & \\
\hline Femmes & & 115233040 \\
\hline Racisme & FN/immigrés: & 672232 \\
\hline & Arabes & 112528 \\
\hline & Racisme général & 1238 \\
\hline Évènements & Cannes & 456910 \\
\hline & Césars & 13141617181920 \\
\hline Auto-dérision & & 320 \\
\hline TF1 & & 2931344248 \\
\hline Actualités & Tokyo & 3546 \\
\hline & Politique & 816212226283845 \\
\hline & Ben Laden & 217 \\
\hline & DSK & 346 \\
\hline & Apple & 263646 \\
\hline Personnalités & Justin Bieber & 2433 \\
\hline & Autres & 2739434749 \\
\hline Autres & & 3750 \\
\hline
\end{tabular}

Tableau $\mathrm{n}^{\circ} 1$ : Thématiques des Tweets de Mouloud

Le relevé de ces thématiques est intéressant en ce qu'il nous informe sur les différentes contraintes qui pèsent sur les productions de l'auteur.

Le discours médiatique dans lequel elles sont effectuées invite à considérer les tweets relevant des catégories «événements 》, «actualité » et " personnalités » comme conditionnées pour partie par ce discours, comme en témoignent les exemples suivants :

6 Les plus grandes fêtes n'étaient pas à Cannes mais au siège du FN, où Marine Le Pen a chauffé le dancefloor pour fêter la mésaventure de DSK.

16 Le césar du meilleur scénario original est attribué à Michèle Alliot-Marie pour L'Avion.

33 Justin Bieber a joué à Bercy devant seize mille fans. Biactol et Clearasil ont fait leur chiffre d'affaires en un soir.

D'autres catégories relèvent davantage du support (magazine $B e$ ), comme les tweets concernant les femmes (qui incluent les relations hommes-femmes) :

1 Le sexe faible est le sexe masculin. Nous sommes incapables d'accoucher ou de faire deux choses en même temps. Respect les filles.

D'autres sont probablement davantage liés à l'auteur, à ses gouts, et à ses origines ou spécificités, donc plus généralement à son style :

7 Les immigrés coûtent 47,9 milliards d'euros en protection sociale. Ils en rapportent 60,3 en cotisations? Ils ne volent donc pas le pain des Français, mais paient le café.

34 Avec son come-back sur TF1, Johnny a fait moins d'audience que Patrick Sébastien sur France 2. Les années bonheur ont battu les années toxine botulique.

20 Et, pour finir, je remets le césar de la personne qui n'était pas présente dans la salle à @ mouloudachour.

Les thématiques présentes dans ces 50 tweets qui constituent notre corpus d'analyse peuvent correspondre à des paramètres distincts :

1. le discours de $B e$ s'inscrit dans un positionnement relatif au public féminin, dans le cadre d'un magazine hebdomadaire, qui rejoint le discours d'actualité et politique. Les tweets à propos des 
femmes ou des relations hommes-femmes s'inscrivent pleinement dans ce positionnement, alors que l'aspect actualité est plus lié au support, et l'aspect politique à l'intertextualité.

2. Le style de l'auteur, que nous considérons d'une manière très générale, conduit à d'autres productions telles que celles sur le racisme, TF1 et l'auto-dérision, bien qu'il y ait bien sûr pas de frontière étanche entre ce style et le discours identifié précédemment. Comme indiqué au point 1 , ce style s'articule au positionnement de l'auteur, rejoignant le concept de formation discursive.

3. Cette «perméabilité » entre les contraintes exercées par le discours et celles qui sont exercées par le style est particulièrement présente pour les événements (cinématographiques par exemple) qui intéressent l'auteur, ou encore les tweets politiques ou sur le racisme qui concernent diversement les deux niveaux d'analyse.

Après avoir travaillé sur les aspects sémantiques de ces tweets, nous proposons d'en étudier davantage la forme, afin de voir si le repérage du «poids» des différents facteurs envisagés peut également se transposer au niveau de l'expression.

\section{4 « Grammaire » du tweet : format et style}

Comme nous l'avons indiqué, un tweet doit comporter au maximum 140 caractères. Dans cet exercice, l'auteur excède parfois légèrement (pouvant aller jusqu'à 170/180 caractères), mais il maintient toujours une brièveté qui est caractéristique de cette rubrique. D'une certaine manière, le tweet de Mouloud peut s'apparenter à une forme sentencieuse. Anscombre (1994) en distingue deux types, selon les auteurs qui en sont à l'origine : elles ont un auteur si leur énonciation les présente comme l'opinion ou le jugement d'une communauté linguistique particulière, qui n'est pas la totalité de la communauté linguistique correspondante. La maxime ou la sentence, par exemple, sont perçues comme ayant un auteur spécifique, même s'il n'est pas nommément connu. Cet auteur est l'énonciateur du jugement général délivré, et il l'appelle l'énonciateur premier.

À cet égard, 18 des 50 tweets comprennent des marques de la $1^{\text {ère }}$ personne du singulier, manifestant explicitement la source du point de vue, comme dans :

\section{J'ai eu la chance de voir le vrai vainqueur du Festival de Cannes : Franck Provost}

36 L’iPad 2 est sorti. Si vous savez à quoi ça sert, envoyez un mail sur mon iPad I.

D'autres ne font pas explicitement mention de cette $1^{\text {ère }}$ personne, mais témoignent de marques de subjectivité ou d'humour caractéristiques de l'auteur, comme dans :

41 La vidéo est explicite : John Galliano est prêt pour la facho week. Il présentera sa collection automne/Hitler.

17 Le césar du meilleur acteur a été attribué à Mouammar Kadhafi pour son rôle dans Kadhafou,

22 Après l'échec du débat sur l'identité nationale, l'UMP lance un débat sur l'islam. Le FN risque d'attaquer pour plagiat.

Dans le tweet 41, «facho » relève d'une certaine stylisation, pour parodier fashion-week, qui légitime ensuite le jeu de mots entre hiver et Hitler. Dans le 17, Kadhafou révèle par l'humour de la suffixation le point de vue de l'auteur. Enfin, dans le 22, c'est l'association entre FN et plagiat qui indique le parti pris de l'auteur dans cette mise en relation.

D'autres tweets ne permettent pas d'identifier directement l'auteur. Ils s'apparentent alors plutôt à des phrases génériques. Rappelons, comme l'explique bien Anscombre, que les phrases génériques peuvent être typifiantes a priori, du type Les voitures ont quatre roues : elles s'opposent d'une part aux phrases analytiques (comme Les baleines sont des mammifères), d'autre part aux phrases typifiantes locales. Ainsi, avec : 
31 En passant à l'heure d'été, on a perdu une heure. C'est comme si on avait tous regardé Carré Viiip en même temps.

l'auteur donne l'impression d'une certaine généricité, en typifiant un des éléments, en l'occurrence « Carré Viiip ». Cela peut aller à un degré davantage affirmé de généricité, comme dans :

47 Quand tu te reconnais dans les paroles d'une chanson de David Guetta, ça signifie que l'heure est grave.

En effet, la structure «Quand... ça signifie que » donne une grande force énonciative à ce tweet, en lui conférant une valeur d'évidence à partir d'un «tu » générique.

Une certaine binarité sémantique caractérise également les tweets de Mouloud, ce qui pourrait les rapprocher des proverbes. Par exemple, le tweet suivant fonctionne sur le mode information>conclusion, sans que l'enchaînement ne se fonde sur le « contenu » informatif du premier membre :

\section{Le single du prochain album de Carla Bruni aurait été volé. Il y a donc une justice} sur Terre!

On retrouve dans certains tweets la convocation d'un topos ou stéréotype (nous ne nous engageons pas dans la discussion terminologique), ici relatif à «album de Carla Bruni » (stéréotype qui pourrait s'énoncer comme La musique de Carla Bruni est néfaste).

Dans « Parole proverbiale et structures métriques », Anscombre (2000) concède que la thèse « bipartite + rime ou isosyllabique » à propos des proverbes est trop forte, et repose sur l'erreur du parallélisme logicogrammatical (qui verrait le proverbe comme une structure «P est un argument pour $Q$ »: en fait il s'agit d'une binarité sémantique que rien n'oblige à représenter par une forme binaire.). D'ailleurs, en ce qui concerne le critère de la rime, il démontre qu'il s'agit en fait de structures rythmiques. Les proverbes sont donc des occurrences de certaines configurations rythmiques, mais il n'y a aucune raison pour qu'un seul schéma prosodique soit à l'œuvre dans les proverbes (chaque époque en privilégie une). Certains des tweets qui constituent notre corpus répondent à cette définition plus souple du proverbe, comme :

42 Le soir des Victoires de la musique, je me suis senti un Français comme les autres : je n'ai pas pu regarder.

On a en effet deux paries distinctes, avec une relation de conclusion représentée par le signe de ponctuation «:». D'autres ont même une forme ternaire, dont le rythme joue pourtant un rôle fondamental :

29 Je n'avais pas la télé cette semaine. Du coup j’ai loupé Enquête exclusive. Du coup, j'ai moins peur des gens.

Ce rythme donne à la fois un effet d'attente, et double le fonctionnement de la chute avec un premier membre en «du coup » qui produit déjà un certain effet, qui accentue la factualité revendiquée, et qui crédibilise la conclusion finale « j'ai moins peur des gens ».

Pour finir, et compléter ces éléments, nous avons pris en compte les spécificités stylistiques du corpus repérées par le logiciel Cordial, dont les principales sont les suivantes :

Indications stylistiques :

Les expressions figées sont nombreuses.

On relève une proportion de noms propres importante.

Le nombre de phrases par paragraphe est très réduit.

Les phrases ont une structuration grammaticale simplifiée.

Ce texte offre un niveau d'abstraction très élevé.

Le langage utilisé est efficace mais peu descriptif. 
Ce texte comporte une proportion assez élevée de mots argotiques ou populaires. Ce texte comporte une proportion réduite de mots ou tournures familières.

La proportion très réduite d'adjectifs de ce texte indique une volonté d'objectivité et de non-description. La proportion de verbes, inférieure à la moyenne, dénote un style plutôt descriptif

Il nous semble possible de spécifier pour chacune le facteur principal :

Style de Mouloud

Expressions figées

Mots argotiques ou populaires

Tournures familières

\section{Contrainte du format}

Nombre de phrases réduit

Structuration simplifiée

Langage efficace $\underline{\text { Genre de discours }}$

Proportion de noms propres

Niveau d'abstraction

Volonté d'objectivité

Notons que ceci peut nous donner une indication relative à la partie $n^{\circ} 2$ : la structuration simplifiée et le langage efficace liés au format pourraient avoir une portée sur le choix des thématiques, puisqu'il faudrait que ces thématiques soient porteuses de simplifications ou enchaînements efficaces. Ainsi, le format interviendrait sur les thématiques, pas directement sur la nature de celles-ci, mais plus d'un point de vue stratégique, pour la mise en cohérence avec cet aspect du format.

Avec les parties 2 et 3 , nous avons vu que le corpus constitué par ces 50 tweets manifeste une certaine hétérogénéité, puisque sa sémantique comme son style dépendent de la mise en oeuvre des différents composants que sont le discours, le style et le format. Aussi, pour en saisir la portée théorique, nous proposons maintenant de procéder à une analyse réflexive pour contribuer à une ébauche théorique synthétique.

\section{Analyses réflexives pour la linguistique du texte}

À travers le traitement sémantique et stylistique du corpus d'étude, nous avons pu mettre en évidence l'interaction dynamique des composants que nous avions identifiés préalablement comme structurant la textualité. Une confrontation de ces résultats à des propositions en sémantique des textes ou linguistique textuelle nous semble porteuse afin de dégager plus clairement le fonctionnement de notre corpus, et par là-même de la textualisation en général.

\subsection{Interaction des niveaux de composition}

Pour Rastier (2001: 42), «la sémantique des textes a notamment pour objectif de décrire cette interaction, selon les quatre ordres de la description linguistique : paradigmatique, syntagmatique, référentiel et herméneutique ». Chacune peut connaître trois degrés de systémacité, par rapport à la langue, aux normes sociolectales des discours et genres, et aux normes idiolectales des styles. Selon Rastier, le style a le mérite de poser synthétiquement deux problèmes que la linguistique se doit d'affronter : celui des normes idiolectales, et celui des caractères esthétiques d'un texte.

En effet, avec les théories modernes du génie, « la notion de style en vient à résumer les particularités d'un auteur, comme si le génie créait son genre propre, en transcendant les genres traditionnels » (Ibid. : 169), puis la conception individualiste du style l'emportera définitivement avec l'esthétique romantique ${ }^{4}$.

Concernant l'interaction des concepts que nous envisageons, nous trouvons donc des éléments de réponse chez Rastier, qui synthétise d'ailleurs ses réflexions sur dialecte/sociolecte/idiolecte en indiquant qu' " un texte se rattache à une langue par son dialecte, à un sociolecte par son genre et son discours, à un idiolecte par son style» (Ibid. : 179), et « de la même manière que l'on peut définir un genre comme une interaction sociolectale entre composantes, on peut définir un style comme une interaction idiolectale 
entre composantes » (Ibid. : 180). Mais ces catégories ne sont néanmoins pas étanches, comme les exemples du corpus cités l'illustrent. En effet, Les Tweets de Mouloud ont révélé une corrélation des plans envisagés, que ce soit au sein d'une rubrique (et certains tweets relèvent alors davantage d'une composante ou d'une autre) ou au sein d'un même tweet.

Le recours à l'énonciation nous semble pertinent afin de considérer ces mécanismes de sémiosis textuelle. Dans les travaux d'Adam (voir par exemple Adam, 2004) l'énonciation est considérée dès la discrimination des unités d'analyse :

\begin{abstract}
L'unité minimale que nous adopterons sera la proposition énoncée. Nous choisissons de l'appeler "proposition énoncée » pour souligner le fait qu'il s'agit, d'une part, d'une unité résultant d'un acte d'énonciation et, d'autre part, d'une unité liée, c'est-àdire constituant un fait de discours et de textualité. (Adam, $2004: 50$ ).
\end{abstract}

De fait, l'inclusion du texte et de son organisation dans l'interdiscours et, par contrecoup, celle de la linguistique textuelle dans le champ englobant de l'analyse des discours, permet de prendre en considération l'interaction sociodiscursive de toute production. Si ces considérations rejoignent celles de Rastier à propos des genres, elles mettent la théorisation des pratiques discursives, dans leur diversité, au centre de l'analyse :

\begin{abstract}
les trois pôles du «thème» (sémantique), du «style» (texture des agencements microlinguistiques) et de la « composition » (structure compositionnelle) doivent être inscrits dans la plus vaste théorie des niveaux d'analyse. [...] Les genres possèdent certes un noyau normatif relativement stable et contraignant pour l'énonciateur, mais néanmoins plus souple et plus plastique que les formes de la langue. En mettant en parallèle le couple de rang microlinguistique que forment la grammaire et le style et le couple de rang macrolinguistique du genre et du texte, je suis tenté de dire que le style est à la grammaire ce que le texte est au genre, c'est-à-dire situé potentiellement dans la zone de variation du système, complémentaire de sa zone normative, représentée par les constantes de plus haute fréquence » (Adam, $2004: 93$ ).
\end{abstract}

Si nous essayons de représenter l'interaction des différents composants identifiés par une schématisation, nous pouvons selon nous élaborer ceci :

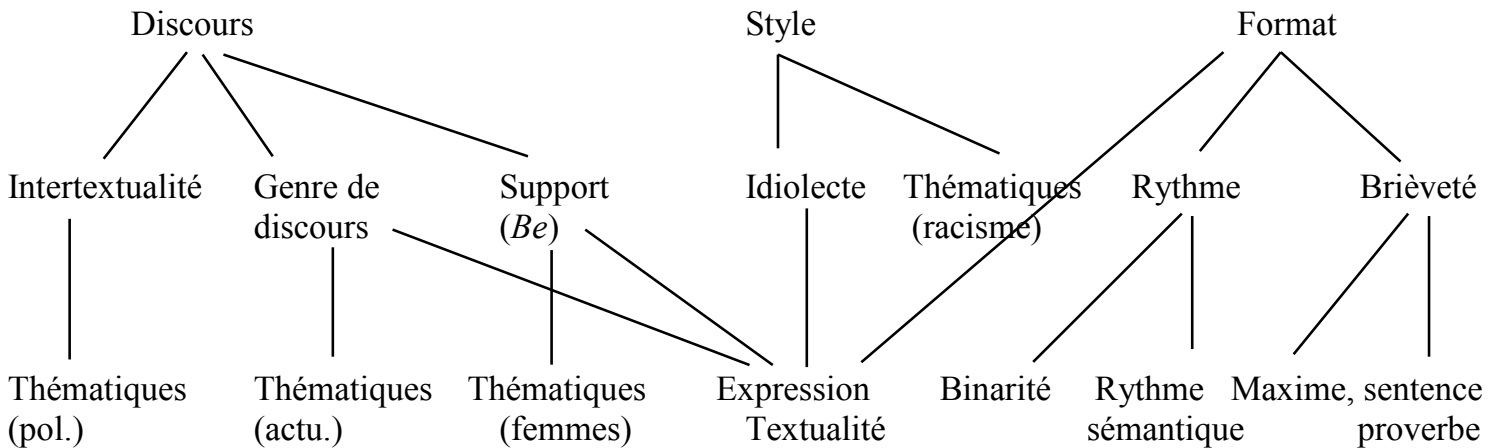

Schéma $\mathrm{n}^{\circ} 1$ : interaction des composants de l'analyse (discours, style et format)

Cette synthèse masque partiellement la complexité du phénomène (son dynamisme), mais montre en particulier que le plan de l'expression ne relève pas du simple style, mais fait interagir des composants du discours et du format. De même, le style et le format ne sont pas de pures formes, puisqu'ils peuvent conduire à l'apparition de thématiques (racisme pour le style par exemple, thématiques propices aux enchaînements pour le format). Aussi, une réflexion sur les plans du contenu et de l'expression nous semble permettre de résoudre le problème que nous avons posé à propos des niveaux de structuration du texte, autour du discours, du style et du format. 


\subsection{Corrélations entre plans du contenu et plan de l'expression}

Pour Rastier (2011 : 42), « Les corrélations entre plans du contenu et de l'expression [...] sont cruciales pour la sémiotique des textes, car elles permettent d'aborder la question de la sémiosis textuelle ». Dans l'agenda dressé par Rastier, la sémantique des textes en corpus met l'accent sur deux complémentarités générales : celle des niveaux de langage ou plans de description (morphologie, syntaxe, sémantique) et celle des paliers d'organisation et de complexité (mot, phrase, texte, intertexte).

Ces aspects rejoignent également les postulats de la grammaire du sens de Charaudeau (voir par exemple Charaudeau, 2001a). En effet la grammaire du sens est caractérisée par trois options :

1. C'est une grammaire de l'énonciation, car la langue y est décrite « du point de vue de catégories qui correspondent à des intentions de communication (le sens), en mettant en regard de chacune d'elles les moyens (les formes) qui permettent de l'exprimer ». La langue est décrite à partir des opérations conceptuelles que fait le sujet parlant quand il communique, et non à partir des catégories morphologiques :

Cela conduit à opérer un regroupement des catégories de formes autour de différentes intentions de communication qui font éclater la structure de la grammaire morphologique. Par exemple, les adverbes de quantité, les adjectifs indéfinis, les déterminants au pluriel (ainsi que le partitif) et des expressions diverses seront regroupés sous la catégorie "quantification"; les adjectifs qualificatifs, certains compléments de nom, certains adverbes, ainsi que les propositions relatives, participes et gérondives seront regroupés sous la catégorie "qualification".

2. C'est une grammaire des effets de discours : cette grammaire montrera que les formes qui servent à les exprimer produisent des effets de sens particuliers selon qu'elles sont employées dans tel ou tel contexte, dans telle ou telle situation. Les catégories linguistiques sont au service du discours et « parler correspond à un enjeu social qui consiste d'une part à s'ajuster à des normes d'usage, d'autre part à jouer avec ces normes. Parler n'est plus affaire d'esthétique mais d'ajustement, d'adéquation et de stratégie » (Ibid.).

3. Enfin, c'est une grammaire pour réfléchir, à partir d'explications sémantiques qui consistent à expliquer les différences de sens entre différentes façons d'exprimer une même intention.

L'usage de cette grammaire permettrait de prendre en compte la définition du genre par Rastier ou Adam par exemple, en reliant expression et contenu dans un même mouvement. Ceci étaye également l'hypothèse d'une corrélation entre les visées discursives et la matérialité linguistique (Charaudeau, 2001b ; Longhi, 2011).

Pour en rendre compte de manière plus systématique, nous avons utilisé le logiciel Tropes, pour une étude plus quantitative de notre corpus. Tropes est un logiciel d'analyse sémantique ou de fouille de textes qui fonctionne sous Microsoft Windows. Ce logiciel a été initialement développé (en 1994) par Pierre Molette et Agnès Landré, sur la base des travaux de Rodolphe Ghiglione. Parmi les fonctions et outils d'analyse de textes proposés par Tropes, ceux qui nous intéresseront sont le diagnostic du style du texte, la catégorisation des mots-outils, et l'extraction terminologique. Tropes utilise des techniques de classification sémantique et de génération de thesaurus qui se rapprochent de la linguistique anglosaxonne, en particulier des travaux de John Lyons. Sa particularité est d'exploiter activement l'Analyse Propositionnelle du Discours (APD) et l'Analyse Cognitivo-Discursive (ACD) de Rodolphe Ghiglione. Surtout, et c'est ce qui justifie son usage dans la problématique qui lie les genres de discours à la grammaire, Tropes s'est aussi inspiré de la Grammaire du sens et de l'expression de Patrick Charaudeau pour diagnostiquer le style du texte.

Nous ne présentons pas en détail tous les résultats, mais pointons des aspects qui sont reconnus comme étant spécifiques de ce corpus. D’un point de vue général, le style est identifié comme plutôt énonciatif, avec une prise en charge à l'aide du « Je ».

Plus précisément maintenant, certains connecteurs et formes de modalisation semblent caractéristiques : 


\begin{tabular}{|l|l|l|}
\hline * Connecteurs : & & * Modalisations : \\
\hline Condition 7.9\% (3) & & Temps 19.3\% (11) \\
\hline Comparais on 10.5\% (4) & & Négation 22.8\% (13) \\
\hline Temps 21.1\% ( 8) & & \\
\hline
\end{tabular}

Tableau $\mathrm{n}^{\circ} 2$ : connecteurs et modalisations caractéristiques

Les Tweets de Mouloud présentent donc des indications de condition, comparaison, temporalité ou négation qui sont en cohérence avec les aspects rythmiques et sémantiques identifiés préalablement. L'auteur joue en effet sur des effets de contradiction, de contextualisation, ou des jeux d'hypothèses, qui créent les structures décalées et humoristiques. En outre, ceci confirme l'hypothèse de l'identification d'un éthos polémique, basé sur la comparaison, la négation, voire la condition. Le repérage des pronoms personnels est également caractérisant de notre corpus :

\begin{tabular}{|l|l|l|}
\hline * Pronoms sg. : & & Pronoms plur. \\
\hline "Je" 50.0\% (38) & & "Nous" 3.9\% (3) \\
\hline "Tu" 7.9\% ( 6 ) & & "Ils" 5.3\% (4) \\
\hline "On" $10.5 \%(8)$ & & \\
\hline
\end{tabular}

Tableau $n^{\circ} 3$ : pronoms caractéristiques

En effet, nous avons une très grande présence du «Je», appuyant la proximité avec les formes sentencieuses, mais le «On» a également un pourcentage élevé, ce qui témoigne de la généricité d'une partie du corpus également.

Avec 31,4\% d'adjectifs numériques, le corpus semble aussi se distinguer par la qualification quantitative, ce qui est cohérent avec le principe de rapporter des éléments factuels et d'actualité ; les chiffres sont en outre mentionnés pour faire ressortir le caractère ludique ou étonnant de certaines informations. Ceci est également en cohérence avec l'intertextualité du discours politique, qui peut user d'une simplification manichéenne des choses.

Le lien entre l'expression et le contenu est donc saisissable par ces quelques exemples de Tropes, qui montrent que les catégories grammaticales participent du fonctionnement signifiant général. Afin de compléter ces indications, nous avons ensuite utilisé l'analyse morphosyntaxique Cordial ${ }^{5}$. Ce qu'il est intéressant de noter, ce sont les comparaisons avec les autres genres de discours. En effet, concernant les types grammaticaux, nous pouvons noter que les comparaisons établies par Cordial avec les autres genres montrent que notre corpus est très atypique : pour chaque catégorie grammaticale, si l'on compare ce texte aux corpus littéraire, technique, journalistique, juridique et commercial, Cordial fournit les résultats suivants :

Proportion de substantifs : il peut être situé entre le corpus des ouvrages à visée commerciale et le corpus des ouvrages à visée commerciale (répétition produire par le logiciel).

Proportion d'adjectifs : il peut être situé entre le corpus des ouvrages journalistiques et le corpus des ouvrages à visée commerciale.

Proportion de pronoms personnels à la première personne : il peut être situé entre le corpus des ouvrages littéraires et le corpus des ouvrages à visée commerciale.

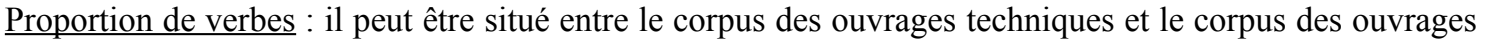
à visée commerciale.

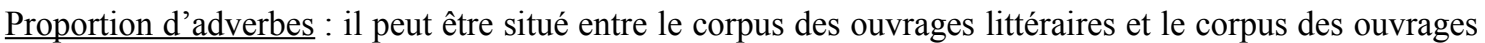
à visée commerciale.

Proportion de pronoms personnels à la seconde ou troisième personne : il peut être situé entre le corpus des ouvrages littéraires et le corpus des ouvrages à visée commerciale. 
Ce qu'il est intéressant de noter, c'est que ce corpus se distingue des autres genres recensés par le logiciel (les proportions de chacune des catégories montrent une originalité du corpus relativement aux autres), mais également que selon les critères il se rapprocherait plutôt des ouvrages à visée commerciale, présents dans chacune des comparaisons (alors que le $2^{\text {nd }}$ terme de comparaison varie). Il est éloigné du discours journalistique si on le caractérise par les parties du discours, ce qui indique que du point de vue de ce type de structuration, le format a un «poids » plus important que le discours, le style participant peut être de cette même élaboration. Même si les considérations formelles sur le genre posent des problèmes (Maingueneau, 2004), ces résultats restent néanmoins intéressants pour contraster notre corpus avec des corpus représentatifs et voisins.

\subsection{Structuration textuelle et portée discursive}

En proposant une description qui mette en interaction les concepts de discours, de style, et de format, nous avons identifié différents niveaux de structuration de la textualité, en rendant compte de leur rendement et de leur interaction. En particulier, nous avons montré que la thématique des tweets est conditionnée par l'intertextualité avec le discours médiatico-politique. Pour finir, nous souhaitons souligner l'intérêt théorique à faire dialogue les approches textuelles avec les approches discursives voire pragmatiques. Ceci est évoqué par Adam qui indique que « la linguistique textuelle doit prioritairement se situer par rapport à deux domaines disciplinaires proches : l'analyse du discours et la pragmatique. » (2004 : 17). Selon lui, aux contraintes générales qui mènent des propositions à l'unité texte, il faut ajouter des contraintes qui sont, à la fois, celles de l'interaction en cours, avec ses paramètres situationnels et psychosociaux, celles de la langue choisie et celles enfin des genres discursifs. Ainsi, cette méthode d'analyse textuelle s'inscrit également dans une approche pragmatique, puisque l'auteur franchit «les frontières classiques du signe, de la proposition ou de la phrase pour aborder les produits naturels de l'interaction langagière, la linguistique textuelle ne procède pas à une simple extension transphrastique des limites du domaine linguistique » (Ibid. : 27). Il s'agit alors de réunir la linguistique transphrastique et la linguistique phrastique de l'énonciation, puisqu'en linguistique textuelle, la séparation du textuel et du discursif est essentiellement méthodologique, et l'évolution de la linguistique textuelle en ferait une pragmatique textuelle.

Le style est lui même problématisé d'une manière originale, et nous suivons en cela Garric et MaurelIndart $(2011: 8)$ :

Nous abordons cependant avec elle [la notion de style] un champ de recherche loin d'être caractérisé par l'unité, comme le montre A. Petitjean (2010 : 245) en concluant le panorama qu'il dresse sur les études du style par ces mots : « le style peut prendre différentes formes de conceptualisation, selon le statut, collectif ou individuel, qu'on lui attribue et duquel dépend la nature des observables ». La tension entre une «stylistique du singulier », définissant un style individuel d'auteur, et une « stylistique du général » (Combe 2002), prônant un style collectif du genre, engendre des positionnements pluriels et divergents.

Nous avons souligné, à la suite de Rastier, la corrélation entre les plans du contenu et de l'expression. Nous pouvons étendre ce constat, et souligner un lien étroit entre discursivité et textualité, en considérant que la textualité a une portée pragmatique propre non seulement à la situation de discours dans laquelle elle se déploie, mais également en lien avec les formations discursives. On insiste souvent, à la suite de Pêcheux, sur ce qui peut et doit être dit en fonction d'une position donnée, mais on s'intéresse moins à la suite de la formule de Pêcheux, " (articulé sous la forme d'une harangue, d'un sermon, d'un pamphlet, d'un exposé, d'un programme, etc.) », mis entre parenthèses par Pêcheux. Or, ce que nous avons avancé dans cet article, c'est que la forme, dont découle la textualisation, est consubstantielle au dire. Aussi, loin d'être un simple " réceptacle » au contenu à formuler par l'auteur, le tweet de Mouloud relève d' " un énonciateur qui produit un discours selon certaines visées va développer des formes perceptives dans des configurations textuelles, dont l'organisation (linéaire et réticulaire) et la planification motiveront un ancrage générique, selon des configurations énonciatives et discursives particulières » (Longhi, 2011 : $165)$. 


\section{Conclusion et perspectives}

Si nous reprenons les différents aspects relevés au fil de cet article, nous pourrions représenter ainsi l'articulation entre les niveaux de structuration de la textualité et les plans du contenu et de l'expression :

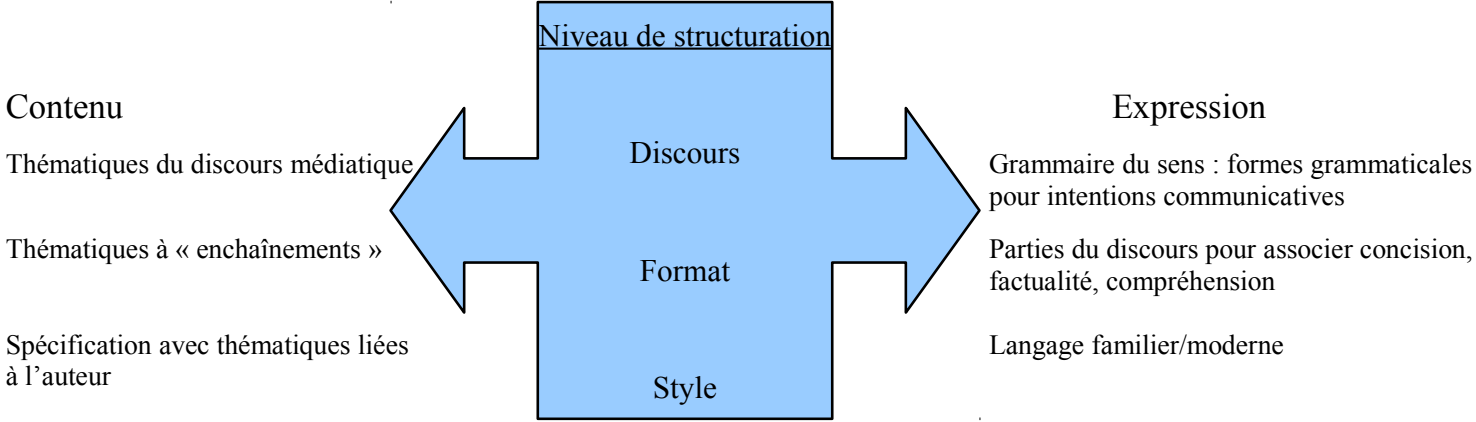

Schéma $\mathrm{n}^{\circ} 2$ : articulation des niveaux de structuration avec les plans du contenu et de l'expression

Finalement, si le corpus des Tweets de Mouloud peut paraître quelque peu trivial en comparaison de corpus littéraires ou médiatiques, il a permis de prendre la mesure des contraintes et des niveaux de structuration de la textualité, qu'elles relèvent du contenu ou de l'expression. En procédant par paliers, nous avons pu saisir à la fois les spécificités des niveaux envisagés - discours, format et styles - nous avons également montré que ces niveaux participent d'un même mouvement, dans lequel contenu et expression se lient pour asseoir les visées discursives propres à la situation d'énonciation. Le lien entre textualité et situation de discours et d'énonciation permet d'entrevoir le rapport entre les marqueurs textuels et les aspects socio-historiques de la production d'un texte, en mettant en rapport la définition d'une formation discursive et ses prolongements stylistiques et les choix de formes et de supports qu'elle propose.

En guise d'ouverture, et pour faire le lien avec des travaux en cours, il nous semble que les résultats obtenus à partir de ce corpus des Tweets de Mouloud pourront être prolongés avec l'usage fait de Twitter dans certains domaines de la communication, comme le discours politique ou médiatique. Nous avons vu récemment que cet outil est utilisé par les journalistes pour rendre compte d'informations (par exemple lors des audiences de DSK, certains journalistes «tweetaient» de l'intérieur de la salle et informaient ainsi leur rédaction, dont les envoyés spéciaux pouvaient alors rendre compte de l'information en temps réel). La déclaration de candidature à l'élection présidentielle de 2012 de Nicolas Sarkozy a été précédée, dans la même journée, de la création d'un compte Twitter, le 15 février 2012 à $8 \mathrm{~h}$ : «Bonjour à tous, je suis très heureux de lancer aujourd'hui mon compte \#Twitter. Merci à ceux qui voudront bien me suivre! - NS ».

Les premières hypothèses de notre travail ${ }^{6}$ sur le discours politique par Twitter confirment la corrélation entre expression et contenu, dans la mesure où la limitation à 140 caractères produit une concision qui s'accompagne d'une sorte d'optimisation sémantique, qui peut aller jusqu'à une intensification signifiante voire un caractère polémique. Pour en rendre compte, voici quelques exemples issus de la campagne présidentielle 2012 :

\footnotetext{
Marie Le Pen

« Hollande est le siamois de Sarkozy »

Hollande, « un champion du mondialisme »

« Hollande et Sarkozy sont interchangeables »

«Hollande est le Chirac de gauche »
} 


\section{François Hollande}

Le scenario est écrit : le président s'est trompé pendant 5 ans, mais justement, ce sera son expérience!

Je vais vous faire une confidence : moi je m'en doutais! A peine entré, il était déjà sortant! \#FH2012

La nouvelle du jour : le président-candidat est désormais candidat-président. Quelle nouvelle! \#FH2012

Il y aurait beaucoup à dire sur ces exemples (nous insistons sur le fait que ce travail est en cours, sur l'analyse du discours politique par Twitter), mais nous voyons déjà une certaine tonalité qui se démarque du discours traditionnel de ces mêmes candidats. Avec les tweets de Marine Le Pen, nous avons un usage de lexèmes originaux : "siamois ", " champion », « interchangeable », et un caractère formulaire avec «Hollande est le Chirac de gauche ». On peut imaginer que ce support produira des énoncés fortement polémiques, dans la mesure où les critiques véhiculées, et leur haut degré, doivent s'incarner par des marqueurs brefs, en particulier dans le lexique (et même si ce lexique est présent dans d'autres contextes (comme « champion » par exemple), la brièveté du tweet en accentue la portée).

Avec François Hollande, on trouve un style et un ton plus humoristique et sarcastique propre à ce candidat, et on remarque que la restriction quantitative ne joue pas directement sur les choix lexicaux, comme dans le cas de Marie Le Pen, mais plutôt sur la textualité, avec des thématisations en début de tweet (« Le scénario est écrit », « Je vais vous faire une confidence » et « La nouvelle du jour », suivis de «:» et de tournures exclamatives qui exploitent le matériau linguistique en jouant sur une plasticité du sens selon les contextualisations ( « expérience » dont le sens attendu « être expérimenté » devient « avoir une expérience », entré/sortant et président-candidat/candidat-président qui critique implicitement la duplicité jouée par Nicolas Sarkozy).

Un autre aspect de l'analyse concernerait l'usage du «\# », qui indique que le mot suivant est « un motclic » (hashtag en anglais), qui devient ensuite un sujet dans Twitter, qui peut être retrouvé (et les sujets deviennent ensuite classés par importance). Ces hashtags constituent donc une forme de modalisation autonymique, qui a une fonction stratégique, doublée d'une dimension performative puisque c'est aussi en créant des sujets que des candidats peuvent se rendre visible (avec \#FH2012, François Hollande tente de se constituer en « sujet ») ou de se constituer comme référence sur un thème donné, comme dans :

Marine Le Pen à \#Toulouse : « Ce n'est pas un \#président de la \#République que nous avons élu, mais un profiteur de la République. »

En effet, Marine Le Pen ne crée par un sujet original, mais se positionne comme référence (puisqu'elle sera effectivement référencée par Twitter) pour les sujets « Toulouse », «président » et « République ».

Le champ reste donc ouvert, et s'il se complexifiera avec la pratique des différents candidats et l'évolution des thématiques de la campagne présidentielle, les jalons théoriques et méthodologiques posés dans cet article, à partir des Tweets de Mouloud, pourront être exploités pour poser le lien entre discours, format et style dans le cadre d'une campagne présidentielle.

\section{Références bibliographiques}

Adam, J.-M. (2004). Linguistique textuelle. Des genres de discours aux textes. Paris : Nathan.

Anscombre, J.C. (1994). Proverbes et formes proverbiales : valeur évidentielle et argumentative. Langue française, $n^{\circ} 102$, p. $95-107$

Anscombre, J.-C. (2000). Parole proverbiale et structures métriques. Langages, $n^{\circ} 139$, p.3-26.

Anscombre, J-C. et Ducrot, O. (1983). L'argumentation dans la langue. Bruxelles : Mardaga.

Charaudeau, P. (2001a). De l'enseignement d'une grammaire du sens. Le Français aujourd'hui, $n^{\circ} 135$. URL: http://www.patrick-charaudeau.com/De-l-enseignement-d-une-grammaire.html 
Charaudeau, P. (2001b). Visées discursives, genres situationnels et construction textuelle. In Analyse des discours. Types et genres, Toulouse : Éd. Universitaires du Sud, URL: http://www.patrick-charaudeau.com/Viseesdiscursives-genres, $83 . \mathrm{html}$

Combe, D. (2002). Stylistique des genres. Linguistique française, vol. 135, $n^{\circ} 1$, p.33-49.

Foucault, M. (1969). L'archéologie du savoir. Paris : Gallimard.

Garric, N. et Maurel-Indart (2011). Introduction. Vers une automatisation de l'analyse textuelle. Texto! URL : http://www.revue-texto.net/index.php?id=2765 .

Longhi, J. (2011). Visées discursives et dynamiques du sens commun. Paris : L'Harmattan.

Maingueneau, D. (2004). Retour sur une catégorie : le genre. In Adam J.-M., Grize J.-B. et Ali Bouacha M. (éds.) : Texte et discours : catégories pour l'analyse, Dijon : Éditions Universitaires de Dijon, p.107-118.

Malrieu, D. et Rastier, F. (2001). Genres et variations morphosyntaxiques. Traitement Automatique des langues, $n^{\circ} 42-2$, p.548-577.

Pêcheux, M. (1975). Les vérités de la Palice. Paris : Maspéro.

Petitjean, A. (2010). Linguistique et littérature, le texte en questions. In Ablali D. et Kastberg-Sjöblom M. (éds.), Linguistique et littérature : Cluny, 40 ans après, Besançon : Presses Universitaires de Franche-Comté, p.197-206.

Rastier, F. (2000). L'accès aux banques textuelles - des genres à la doxa. Texto ! juin 2002. URL : http://www.revuetexto.net/Inedits/Rastier/Rastier_Acces.html

Rastier, F. (2001). Art et science du texte. Paris : P.U.F.

Rastier, F. (2011). La mesure et le grain. Sémantique de corpus. Paris : Honoré Champion.

Sarfati, G.-E. (1997). Éléments d'analyse du discours. Paris : Nathan. 
${ }^{1}$ Nous avons bien conscience du caractère très général de cette caractérisation, nous souhaitons simplement souligner une certaine différence de lectorat entre $B e$ et les magazines cités, même si les frontières de ce lectorat ne sont pas si nettes, ni figées.

${ }^{2}$ À notre connaissance, aucune approche linguistique ou discursive ne s'est encore intéressée aux tweets. Une conférence de M.-A. Paveau, "Genre de discours et technologie discursive. Tweet, twittécriture et twittérature », le 21 octobre 2011, a abordé ces questions (une publication est en cours), soit après la soumission de cet article. Il est donc intéressant de noter que ce type d'écriture commence à susciter de l'intérêt, même si dans notre cas il s'agit de tweets « fictifs ». Notons également que le groupe de travail $\mathrm{n}^{\circ} 7$ du Consortium Corpus Ecrits de l'ILF s'intéresse au thème : "Corpus d'écrits modernes et prise en compte de nouveaux modes de communication (SMS, Blog, courriel...) ». Notre participation à ce groupe permettra peut-être d'introduire Twitter parmi ces nouveaux modes de communication.

${ }^{3}$ Nous avons bien conscience que cette classification, proche de l'analyse de contenu, est déjà une forme de lecture des tweets. La sélection s'est effectuée à partir d'un critère interprétatif facilité par la brièveté des tweets, au regard notamment d'éléments lexicaux, comme nous le verrons dans les analyses.

${ }^{4}$ Ou aussi (Rastier 2001, p.172) : « l'histoire du style est ainsi passée d'une mystique des castes, telle que la reflétait la hiérarchie des genres, à une mystique de l'individu, créateur génial ».

${ }^{5}$ Pour un exemple d'analyse morphosyntaxique avec ce logiciel, voir le travail de Malrieu et Rastier (2001).

${ }^{6}$ Ces éléments étant en cours d'analyse, nous soulignons le caractère d'hypothèse de travail ou d'ébauche de résultats pour les éléments que nous présentons sur Twitter et le discours politique. Il nous semble néanmoins intéressant, dans une perspective textuelle et stylistique, de prolonger le présent travail par quelques exemplifications attestées de tweets liés à la communication politique. Les analyses mériterons d'être approfondies, et prolongées à partir d'un corpus plus vaste et d'une méthodologie mieux affirmée. 